\title{
Connections of the Corticomedial Amygdala in the Golden Hamster. II. Efferents of the "Olfactory Amygdala"
}

\author{
GOLDA A. KFVETTER ANM SARAH S. WINANS \\ Neuroscience Program and Department of Anatomy, The University of Michigan, Ann \\ Arbor, Michigan 48109
}

\begin{abstract}
The anterior cortical (C1) and posterolateral cortical (C2) nuclei of the amygdala are designated the "olfactory amygdala" because they each receive direct projections from the main olfactory bulb. The efferents of these nuclei were traced after stereotaxic placement of $1-5 \mu \mathrm{Ci}$ tritiated proline in the corticomedial amygdala of male golden hamsters. Following survival times of 12 , 24 , or 48 hours, $20 \mu \mathrm{m}$ frozen sections of the brains were processed for light microscopic autoradiography.

Efferents from C2 terminate in layers II and III of the olfactory tubercle and in layer $\mathrm{Ib}$ of pars ventralis and pars medialis of the anterior olfactory nucleus. Fibers from this nucleus also project to layers I and II of the infralimbic cortex and to the molecular layer of the agranular insular cortex. More posteriorly, fibers from $\mathrm{C} 2$ terminate in layer I of the dorsolateral entorhinal cortex, and in the endopiriform nucleus.

From $\mathrm{C} 1$, efferent fibers travel in the stria terminalis and terminate in the precommissural bed nucleus of the stria terminalis and in the mediobasal hypothalamus. Efferents from $\mathrm{C} 1$ also innervate the molecular layer of $\mathrm{C} 2$, the amygdalo-hippocampal area, and the adjacent piriform cortex.

Neurons in both $\mathrm{C} 1$ and $\mathrm{C} 2$ project to the molecular layer of the medial amygdaloid nucleus and the posteromedial cortical nucleus of the amygdala, the plexiform layer of the ventral subiculum, and the molecular layer of the lateral entorhinal cortex.
\end{abstract}

A dual olfactory system was originally postulated on the basis of the anatomical observation that the main olfactory and vomeronasal pathways have no common sites of termination from their origins in their respective receptor epithelia to the terminals of the main and accessory olfactory bulb mitral cells in the piriform lobe (Winans and Scalia, '70; Raisman, '72; Scalia and Winans, '75, '76). It was also suggested that the separation of olfactory and vomeronasal systems is maintained in their projections from the piriform lobe to the diencephalon and telencephalon. Although subsequent anatomical studies of the projections of the piriform lobe have produced evidence that generally supports this concept of segregation, both anatomical and behavioral studies have indicated that the olfactory and vomeronasal systems are not entirely independent. A complementary function between these two systems has been suggested to account for deficits in sexual behavior observed after olfactory and vomeronasal deaf- ferentation in male hamsters (Winans and Powers, '77), and the results of mating behavior studies after brain lesions have focused attention on the amygdala as a possible site for this interaction (Devor, '73; Lehman et al., '78).

The results of anatomical investigations reported here and elsewhere also point to the amygdala as an area of olfactory and vomeronasal integration. Although the vomeronasal amygdala (medial and posteromedial cortical nuclei) does not project to any olfactory areas of the piriform lobe (Kevetter and Winans, '80), these nuclei receive input from the anterior and posterolateral cortical nuclei of

\footnotetext{
This work was part of a disgertation submitted in partial fulfilment of the requirements for the degree of Doctor of Philosophy in Neuroscience, The University of Michigan.

G.A. Kevetter's current address: Marine Biomedical Institute, 200 University Boulevard, Galveston, Texas 77550.

Please gend reprint requests to: Dr. Sarah S. Winans, Department of Anatomy, 5705 Medical Science Il, The University of Michigan, Ann Artor, Michigan 48109.
} 
the "olfactory amygdala," as reported below. The only previous studies that have dealt specifically with the projections from the olfactory amygdala are the recent investigations in the rat and cat by Krettek and Price ('74a, b, c; '77a, b; '78a, b), whose findings differ in several respects from those reported here for the hamster. In general, however, the data from all three species show that the projections of the olfactory amygdaloid areas, like other olfactory bulb recipient areas, send efferents to terminal fields in the telencephalon that are separate from those of the vomeronasal amygdala.

It should be noted that the terminology used by Krettek and Price (78b) for the cortical nuclei in the amygdala that receive a direct projection from the main olfactory bulb differed slightly from the terminology developed by Scalia and Winans ('75), which is used here. The terminology for the anterior cortical nucleus (C1) is the same. Krettek and Price ('78b) called the posterolateral cortical nucleus (C2) by the name "periamygdaloid cortex" because of its resemblance with the adjacent piriform cortex. However, the term "periamygdaloid cortex" has conventionally been used to refer to that part of the piriform cortex adjacent to the amygdala (Lammers, '72). Cytoarchitectural differences (Scalia and Winans, '75; Krettek and Price, '78b) and differences in the projections between $\mathrm{C} 2$ and the piriform cortex (Krettek and Price, '77a) have convinced us to retain the terminology posterolateral cortical nucleus or $\mathrm{C} 2$.

\section{METHODS}

Autoradiographic studies were performed on 50 animals. One to five microcuries of tritiated proline (L-[3,4- $\left.{ }^{3} \mathrm{H}(\mathrm{N})\right]-$ Proline; $25-50 \mathrm{Ci} /$ mmole; New England Nuclear) in 0.01 to 0.05 $\mu l$ physiological saline were injected into one of the four components of the corticomedial amygdala (medial, anterior cortical, posterolateral cortical, or posteromedial cortical nu- clei) via a $1 \mu l$ or $5 \mu$ l Hamilton syringe. Survival time after the injection varied from six hours to six days, with the survival times of most animals being either 12,24 , or 48 hours. Animals were then perfused through the heart with saline, followed by $10 \%$ phosphate-buffered formalin, and the brains were removed and prepared for autoradiographic analysis as previously described (Kevetter and Winans, '81).

\section{RESULTS}

Because the hamster amygdala is a small and compact matrix of nuclei, and because diffusion of the labeled amino acid around the injection site involved more than one nucleus in most experiments, analysis of the data required strict adherence to criteria for both identification of the injection site and determination of the projections from each nucleus. The criteria used have been outlined previously (Kevetter and Winans, ' 80 ). Conclusions about the projections of the nucleus under investigation were based on comparison of results from brains in which the injections all included the target nucleus but had no other areas in common. Brains with injections that did not involve the target nucleus were analyzed as controls. For analysis of the projections of the anterior cortical nucleus (C1), this control group included brains with injections of the posterolateral nucleus (C2), and vice versa. In the case of $\mathrm{C} 1$, the projections reported below were seen in at least ten of the 14 brains examined, and in the case of $\mathrm{C} 2$ all of the projections reported were seen in ten of 13 brains.

\section{Projections of the anterior cortical nucleus}

Fourteen animals had injections that primarily involved C1. The maximum extent of diffusion into areas surrounding $\mathrm{C} 1$ is shown in coronal sections of two representative brains in Figure 1. The needle tracts com-

Abbreviations

AC, anterior commissure

AHA, amygdalo-hippocampal area

AI, agranular insular cortex

$A O N$, anterior olfactory nucleus

AONm, anterior olfactory nucleus, pars medialis

$\mathrm{AONe}$, anterior olfactory nucleus, pars externa

BLa, anterior division basolateral nucleus of the amygdala

BLp, posterior division basolateral nucleus of the amygdala

BNST, bed nucleus of the stria terminalis

$\mathrm{C1}$, anterior cortical nucleus of the amygdala

$\mathrm{C2}$, posterolateral cortical nucleus of the amygdala

C3, posteromedial cortical nucleus of the amygdala

$\mathrm{Ce}$, central nucleus of the amygdala
CPF, piriform cortex

$\mathrm{DB}$, diagonal band of Broca

$\mathrm{E}$; endo, endopiriform nucleus

EC, entorhinal cortex

IL, infralimbic cortex

$L$, lateral nucleus of the amygdala

$\mathrm{M}$, medial nucleus of the amygdala

MOB, main olfactory bulb

OTu, olfactory tubercle

$S$, subiculum

ST, atria terminalis

tt, tenia tecta

VMH, ventromedial nucleus of the hypothalamus 

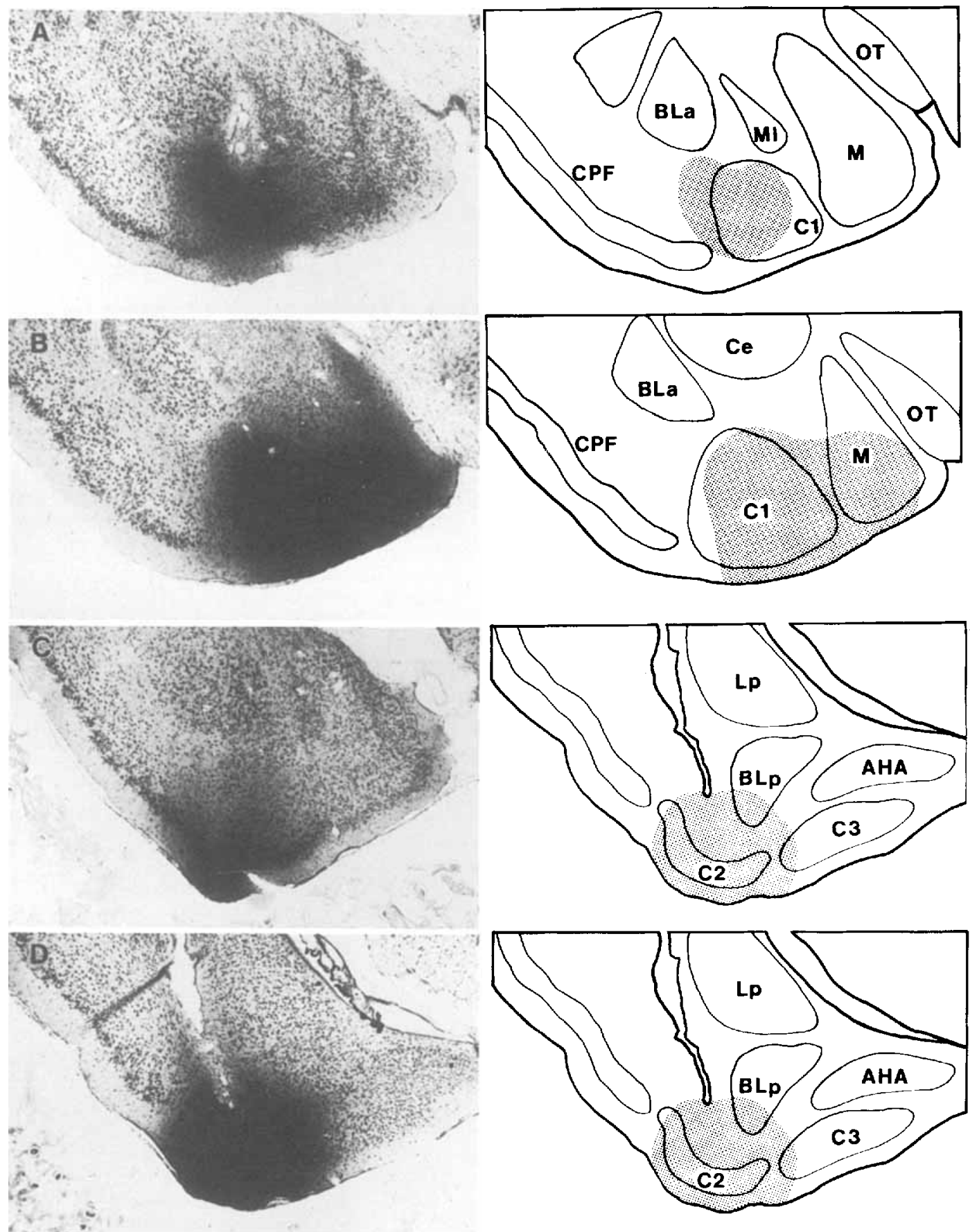

Fig. 1. Brightfield photomicrographs and matching tracings of injection sites centered in $\mathrm{C} 1$ and C2. Area of stippling corresponds to locus of the injection as defined by heavily labeled neurons. $B$ and $D$ show two of the largest injection sites used for this analysis. A. animal $63 ; 5 \mu \mathrm{Ci}$ ${ }^{9} \mathrm{H}$-proline in $50 \mathrm{nl}$ physiological saline; survival time $=\mathbf{2 4}$

hours. B. animal 102; $2 \mu \mathrm{Ci}^{3} \mathrm{H}$-proline in $20 \mathrm{nl}$ physiological saline; survived 24 hours. C. hamster $94 ; 1 \mu \mathrm{Ci}{ }^{3} \mathrm{H}$-proline in $10 \mathrm{nl}$ physiological saline; survived 24 hours. D. animal 93; $2 \mu \mathrm{Ci}{ }^{3} \mathrm{H}$-proline in $20 \mathrm{nl}$ physiological saline; survived 25 hours. 
monly traversed the cortex, corpus callosum, and internal capsule. In some brains the fornix, striatum, lateral nucleus of the amygdala, optic tract, hippocampus, and reticular nucleus of the thalamus were also traversed. The projections from these areas were distinguishable from those of $\mathrm{C} 1$ because the terminal fields arising from projections of the nonamygdaloid areas, in contrast to those from $\mathrm{C} 1$, were inconsistently labeled in brains with large injections of $\mathrm{C} 1$. In addition, Fink-Heimer sections prepared from brains with lesions in the amygdala allowed us to follow fibers emanating from the electrode tracts to their terminal fields. Brains in which the stria terminalis had been damaged were not analyzed.

The projections of $\mathrm{C} 1$ as determined in these 14 brains are shown diagrammatically in Figures 2, 3, and 4. Most of the projections are confined to the piriform lobe, primarily to the amygdala. A heavy concentration of label is seen in layer Ib of $\mathrm{C} 2$ (Fig. 3G-I) and the posteromedial cortical nucleus (C3) (Fig. 5; Fig. $3 \mathrm{H}-\mathrm{K}$ ). In brains in which the medial nucleus is not involved in the injection, silver grains are seen concentrated over the Ib-II junction of the medial nucleus. Terminals are also seen in layer I of the ventral part of the lateral entorhinal area just caudal to C3 (Fig. 3K), in the plexiform layer of the ventral subiculum, and concentrated along the ventral border of the amygdalo-hippocampal area (AHA) (Fig. 3). It was not clear from this material whether the terminal field on the ventral border of AHA represented terminals on the dendrites of AHA neurons or perhaps on those of the dorsal part of C3.

Label is also seen in the piriform cortex adjacent to the injection area. Since the grains are concentrated over the deep molecular layer (Ib) and not uniformly distributed over the area, it appears that this label is due to a projection from $\mathrm{C} 1$ to the piriform cortex, and not to diffusion of label from the injection site.

Projections from C1 to the diencephalon travel in the medial component of the stria terminalis. Some fibers exit at the level of the anterior thalamus and travel ventrally toward the medial preoptic-anterior hypothalamus junctional area. These fibers then travel caudally through the mediobasal hypothalamus to the core of the ventromedial nucleus of the hypothalamus, where they terminate. Other fibers in the stria terminalis continue rostrally to the level of the caudal olfactory tubercle, where they exit and terminate in the medial bed nucleus of the stria terminalis at the medial edge of the anterior limb of the anterior commissure (Figs. 2D; 6).

\section{Projections of the posterolateral cortical nucleus}

Thirteen animals had injections primarily involving $\mathrm{C} 2$. Two representative cases of injections involving $\mathrm{C} 2$ are shown in Figure 1. A schematic representation of C2 projections is shown in Figures 2, 3, and 4.

The needle tract often traversed the cerebral cortex, internal capsule, and corpus callosum. The entorhinal cortex, lateral nucleus, or basolateral nucleus were also involved in some cases. As in the case of $\mathrm{C} 1$ injections, the possible contamination of these areas was considered in analyzing the data.

From C2 some fibers travel dorsally to enter the middle portion of the stria terminalis. Rostral to the decussation of the anterior commissure, many of these fibers leave the stria terminalis and descend between the diagonal band of Broca and the nucleus accumbens to terminate in layers II and III of the medial part of the olfactory tubercle (Figs. 2B, C; See Fig. 8). Terminals are not seen over the cell bodies in the Islands of Calleja. Instead, most of the grains are located on the superior edge of a cell-sparse area above the islands, giving the appearance of a halo over each island in coronal sections. Label in the areas between the islands was sparse. Additional fibers from the rostral extension of the stria terminalis exit ventral to the ependymal cells and course medially through the cells of the cortex to a terminal field at the junction of layers $I$ and II throughout the infralimbic cortex (Fig. 2B). At the level of the anterior olfactory nucleus, fibers that have traveled anteriorly in the intermediate olfactory tract terminate in layer $\mathrm{Ib}$ of pars medialis and pars ventralis of the anterior olfactory nucleus (Figs. 2A; 7).

Fibers from C2 also traverse the external capsule and endopiriform nucleus to terminate in layer I of the dorsal and posterior agranular insular cortices. Some of these fibers appear to terminate in the caudal part of the endopiriform nucleus pars dorsalis.

Additional terminals are concentrated in the "shell" of the posteromedial cortical nucleus of the amygdala, the plexiform layer of the ventral subiculum, and the lateral entorhinal areas (Fig. 3J, K). Label is confined to layers I and III of the lateral entorhinal area and layer I of the dorsolateral entorhinal cortex. 
Lastly, efferents of C2 terminate in layers I and II of the medial nucleus of the amygdala. The pathways taken by many of the fibers that terminate within the piriform area could not be identified.

\section{DISCUSSION}

A comparison of the projections of the olfactory amygdala reported here and projections of the vomeronasal amygdala (Kevetter and Winans, '81) in hamster has shown that the concept of a dual olfactory system is generally valid. The vomeronasal amygdala projects predominantly to other vomeronasal areas (Kevetter and Winans, '81), whereas the olfactory amygdala projects to areas that receive projections from the olfactory cortex (Fig. 4). However, in the hamster both vomeronasal and olfactory components of the rostral corticomedial amygdala, $M$ and $C 1$, project outside of their respective circuits to the ventromedial nucleus of the hypothalamus. In addition, the two systems are interconnected by projections from the anterior cortical nucleus to $\mathrm{M}, \mathrm{C} 3$, and BNST, all vomeronasal pathway areas. The anterior cortical nucleus also projects to AHA of the amygdala and the subiculum. The posterolateral cortical nucleus, like $\mathrm{C} 1$, projects to M, C3, and the subiculum; and in addition, C2 projects to the endopiriform nucleus, which projects to both $\mathrm{M}$ and $\mathrm{C} 3$ in rat (Krettek and Price, '78b). Thus there is substantial input, direct and indirect, from the olfactory to the vomeronasal portions of the amygdala.

Some of the projections ascribed to the individual components of the "olfactory" amygdala in rat and cat (Krettek and Price, '78b) differ from those reported here for hamster. Those projections identified in the hamster that have not been previously reported include connections from $\mathrm{C} 1$ to the medial amygdaloid nucleus, AHA, BNST, and VMH. In contrast, the projection to the nucleus of the lateral olfactory tract from $\mathrm{C} 1$ in the cat (Krettek and Price, '78b) was not seen from either $\mathrm{C} 1$ or $\mathrm{C} 2$ in the hamster. Another distinction between the efferents reported here for hamster and those reported for rat and cat involves the inputs from the olfactory amygdala to the AON and frontal cortex. Whereas in the hamster projections from $\mathrm{C} 2$ terminate in the $\mathrm{AON}$, medial, and lateral prefrontal areas, in the rat and cat the fibers that project to these three areas originate in $\mathrm{C} 1$ (Krettek and Price, '74b, '74c, '77a, '78a; Haberly and Price, '78a). These differences are probably best viewed as topo- graphic variations rather than as significant species differences.

\section{Anterior olfactory nucleus and prefrontal cortical areas}

In the hamster projections from $\mathrm{C} 2$ are seen to the anterior olfactory nucleus and olfactory tubercle, areas that receive a direct projection from the main olfactory bulb (Broadwell, '75a, 75b). As in rat and cat (Krettek and Price, '78a, b), label transported from C2 accumulated in a halo pattern above the islands of Calleja of the medial olfactory tubercle (Fig. 8). Although Haberly and Price ('78a) reported projections from both $\mathrm{C} 1$ and $\mathrm{C} 2$ to the olfactory tubercle in rat, they reported the projection from $\mathrm{C} 1$ to be sparse. It is conceivable that a projection from $\mathrm{C} 1$ to the tubercle was not identified in the hamster because the sparse distribution of terminals could not be reliably distinguished from background.

de Olmos and Ingram described amygdaloid projections to the AON in the rat in 1972, but they did not localize the origin of those projections. In 1978 Haberly and Price ('78a) reported that these projections arise from $\mathrm{C} 1$. In hamster, however, this projection originates in C2; it travels in the parolfactory stria terminalis and terminates in layer $\mathrm{Ib}$ of the pars medialis of the AON (Fig. 7). Evidence from horseradish peroxidase (HRP) labeling of olfactory bulb afferents in the rat (de olmos et al., '78; Haberly and Price, '78a, b) and hamster (Davis et al., '78) demonstrates a projection from AON pars medialis to the bulb, although it may be sparse in the hamster. Thus the input from the olfactory amygdala to the AON may provide an indirect pathway by which the amygdala can modulate activity in the main olfactory bulb. In addition, a direct pathway from $\mathrm{C} 2$ to the olfactory bulb has been identified in the rat (de Olmos et al., '78) and hamster (Davis et al., '78).

Projections from the "olfactory" amygdala also terminate in the infralimbic and agranular insular cortices. Projections from $\mathrm{C} 2$ to layer I of the infralimbic cortex in the hamster follow a pathway similar to that reported by Krettek and Price ('77a) from C1 in the rat. In the cat an additional, though more sparse, area of terminals was found from $\mathrm{C} 1$ to the prelimbic area layer I by Krettek and Price ("77a).

Layer I of the dorsal and posterior agranular insular cortices also receives a projection from C2 in the hamster. As noted above, Krettek and Price ('77a) reported projections from C1 
A

B
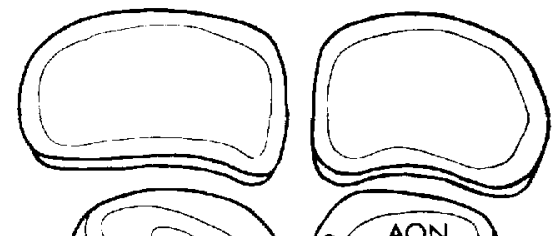

C

D

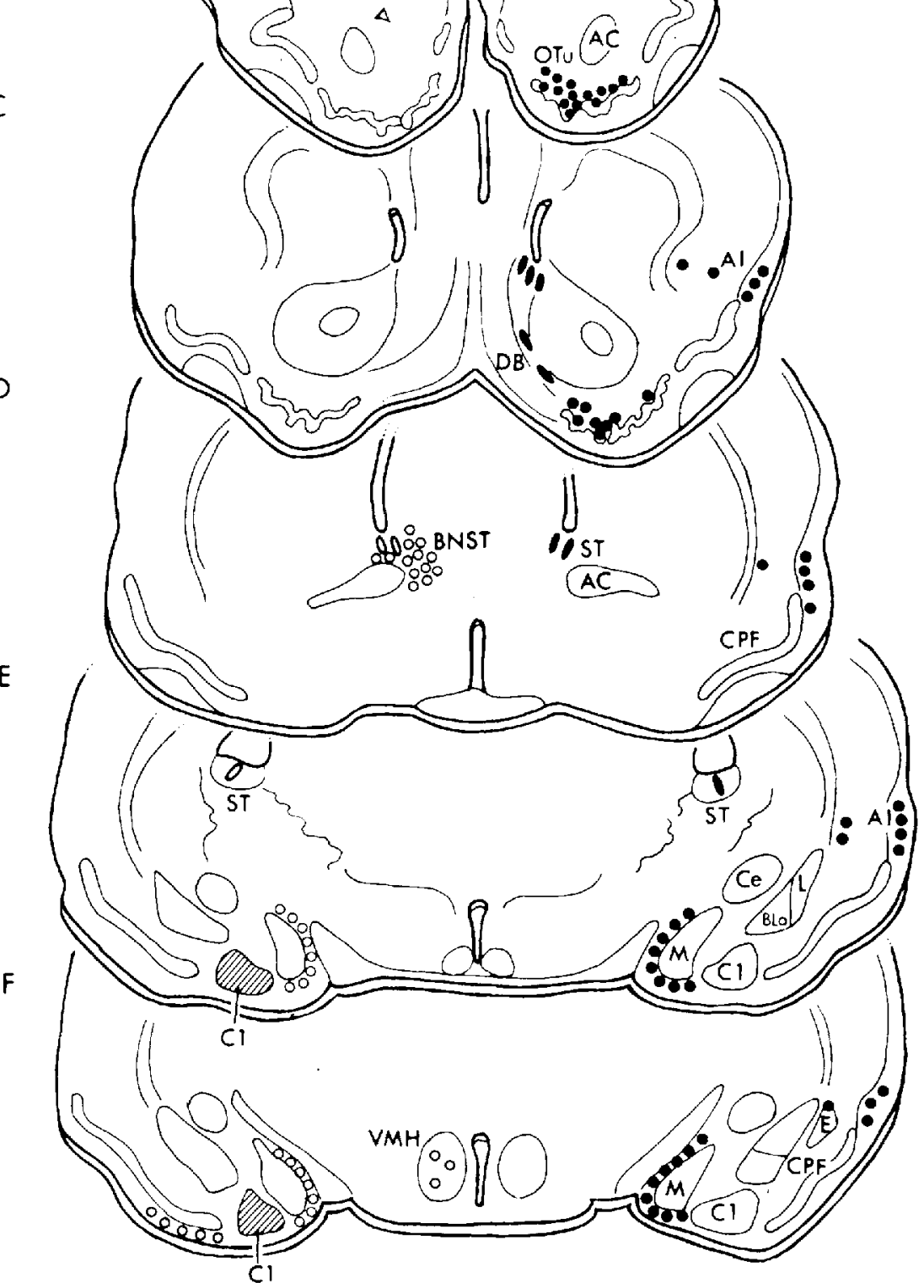

Figs. 2 and 3. Schematic drawings of coronal sections of the hamster brain showing the neuronal projections from the anterior cortical nucleus on the left (open circles) and the posterolateral cortical nucleus on the right (closed circles). 


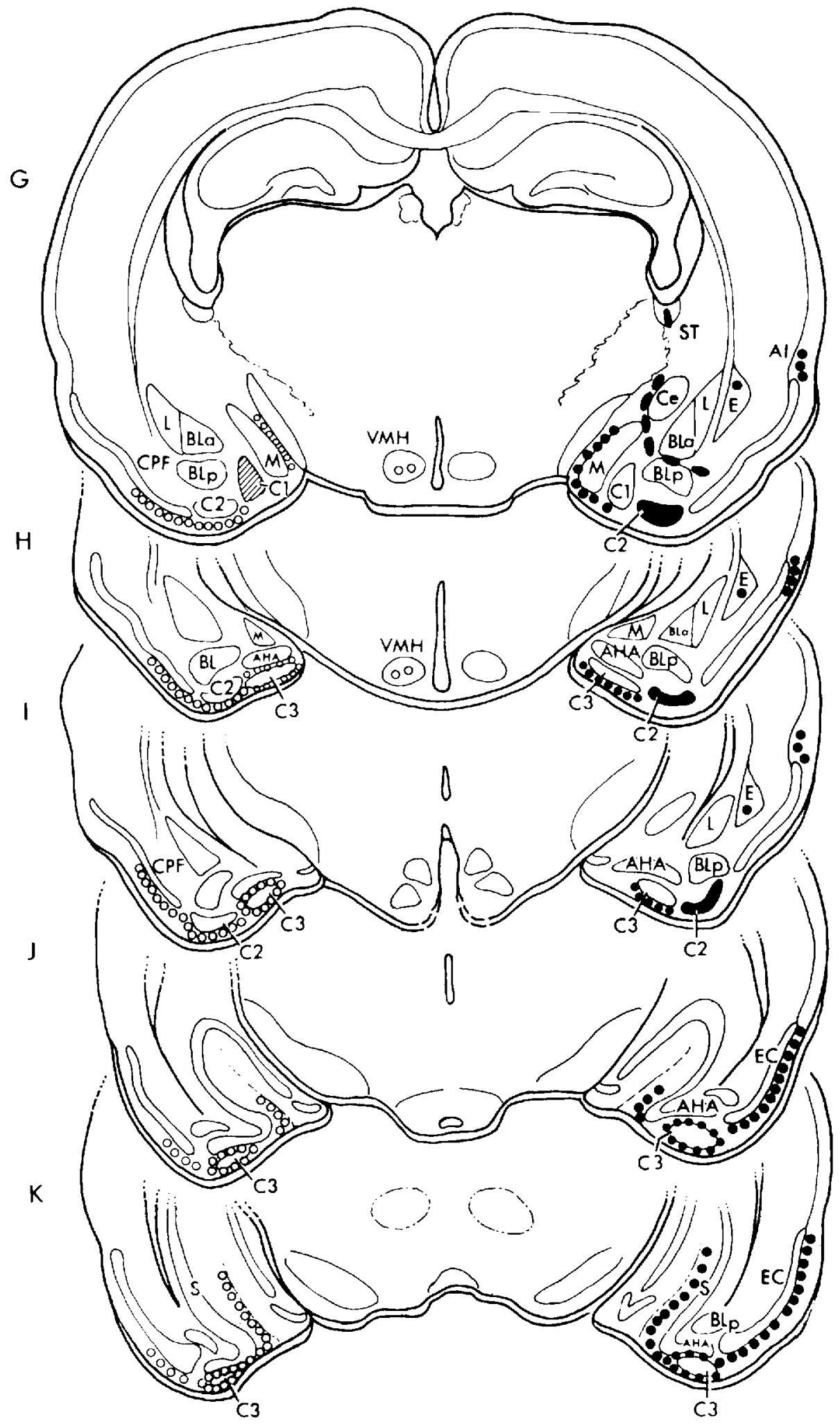

Figure 3. 


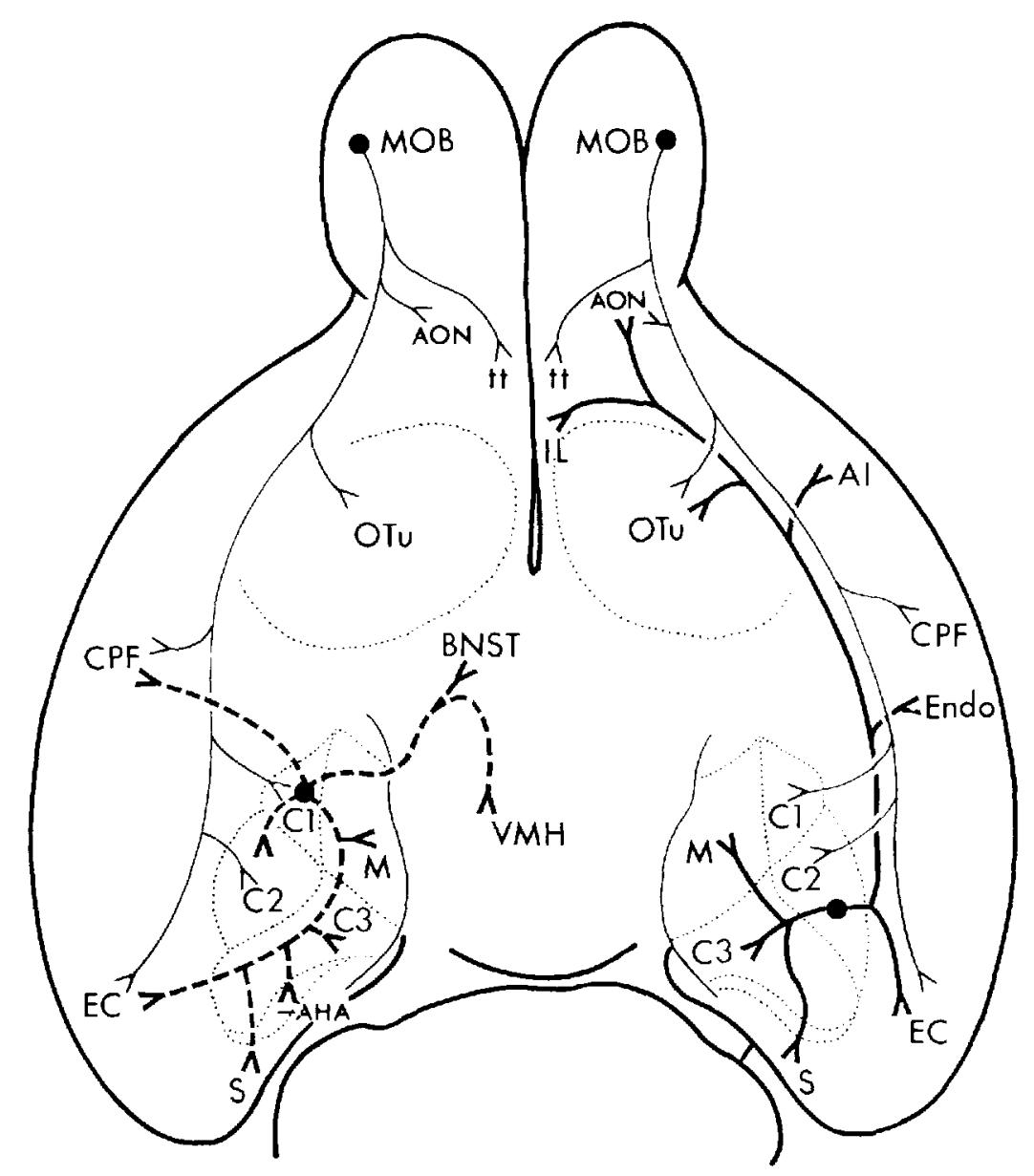

Fig. 4. Schematic diagram of the ventral surface of the hamster brain showing the efferents of the main olfactory bulb (thin solid line), anterior cortical nucleus, C1 (heavy solid line), and posterolateral cortical nucleus, $\mathrm{C} 2$ (dashed line).

to layer I of the ventral and posterior agranular insular areas and perirhinal cortex in rat and cat. In the hamster fibers from $\mathrm{C} 2$ exiting from the external capsule pass through the endopiriform nucleus where some of them terminate. This latter connection, from $\mathrm{C} 2$ to the endopiriform nucleus, is in agreement with the projection from C2 seen in the rat (Krettek and Price, '78b).

\section{Diencephalic projections}

Although many of the projections from the "olfactory" amygdala are to other olfactory areas, the projections from $\mathrm{C} 1$ to the bed nu- cleus of the stria terminalis (BNST) and VMH correspond to vomeronasal pathway connections. No projection to the BNST or VMH from $\mathrm{C} 1$ or $\mathrm{C} 2$ was seen in rat or cat (Krettek and Price, '74a, '78a). In the hamster the terminal field of the projection from C1 to BNST is rostral to the terminals from vomeronasal areas $\mathrm{M}, \mathrm{C} 3$, and $\mathrm{AOB}$, but the projection from $\mathrm{C1}$ to the mediobasal hypothalamus overlaps the diencephalic projection from M. Fibers from both $\mathrm{C} 1$ and $\mathrm{M}$ travel in the medial part of the stria terminalis and terminate in the core of the ventromedial nucleus. Evidence for this projection has also been reported by McBride and Sutin ('77) after HRP studies in the cat. 

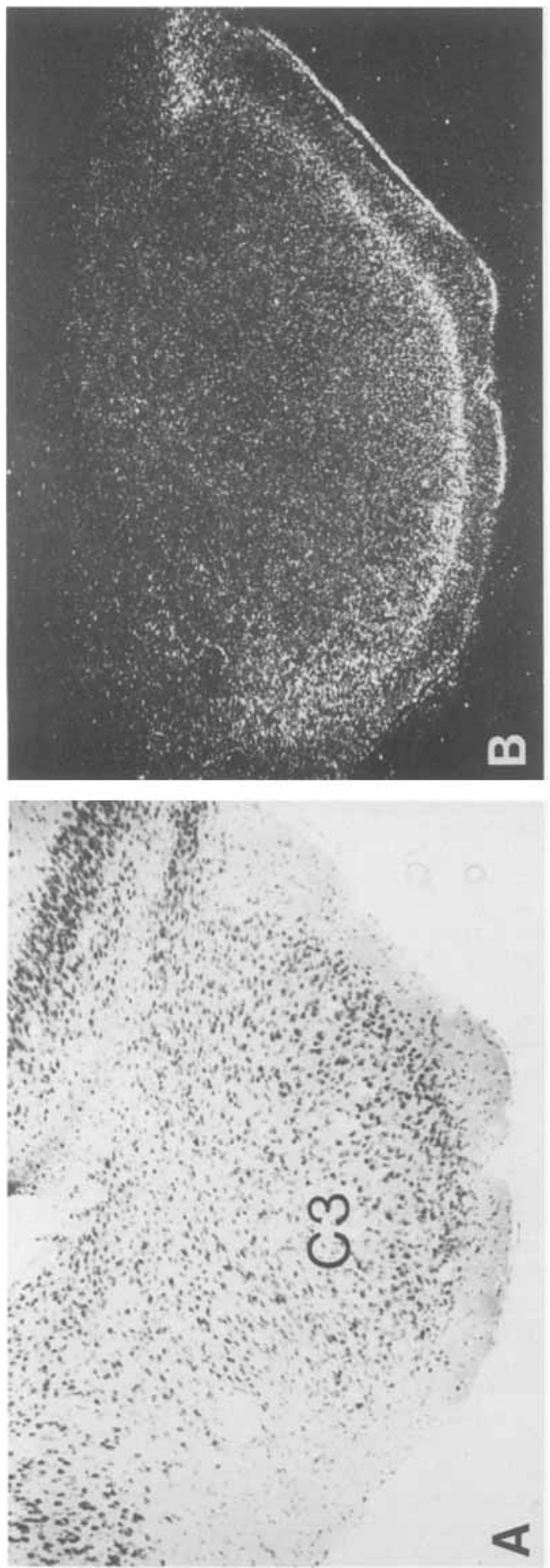

\section{Piriform areas}

Fibers from $\mathrm{C} 1$ travel to $\mathrm{C} 2$, the piriform cortex, and the amygdalo-hippocampal area. Label is observed in layer Ib of the molecular layer of $\mathrm{C} 2$ and the piriform cortex, while the projection to the amygdalo-hippocampal area appears to terminate along the ventral border of this area. Krettek and Price ('78b) noted that the distribution of silver grains associated with the amygdalo-hippocampal area was continuous with grains over the subiculum. Thus the silver grains seen near the amygdalo-hippocampal area may represent fibers that originate in C1 and C2 (and perhaps the medial nucleus) and terminate in the subiculum.

Projections from C1 to the ventrolateral entorhinal area layer Ib-II and from $\mathrm{C} 2$ to the dorsolateral entorhinal area layers I and II were seen in hamster. Fibers collect in the junction between C3 and the amygdalo-hippocampal area, travel along the ventral border of the latter nucleus, and terminate in the dorsolateral entorhinal area. Fibers that terminate in the ventrolateral entorhinal cortex travel in the junction of $\mathrm{C} 3$ and the amygdalohippocampal area and then turn lateral to reach their terminal fields. Krettek and Price (77a) reported a projection from $\mathrm{C} 2$ to the deep parts of layer I, and layer II, in adjacent portions of the ventromedial and ventral parts of the lateral entorhinal area.

In the hamster, $\mathrm{C} 1$ and $\mathrm{C} 2$ both project to the plexiform layer of the ventral subiculum. Fibers travel medially from the medial edge of C3 and the amygdalo-hippocampal area. These findings confirm the projections that Krettek and Price ('77a) described from C2 to the subiculum in the rat and cat.

The intra-amygdaloid connections observed in the hamster provide a basis for interconnection of the olfactory and vomeronasal parts of the amygdala. $\mathrm{C} 1$ and $\mathrm{C} 2$ both send fibers that terminate in $\mathrm{M}$ and $\mathrm{C} 3$. These projections terminate in the molecular layer of $\mathrm{C} 3$ and the junction of layers I and II in the medial nucleus. Further interactions between olfactory and vomeronasal systems may take place in the ventromedial hypothalamus, where projections from $\mathrm{C} 1$ and $M$ terminate over the neuron cell bodies. In addition terminals from

Fig. 5. Posteromedial (C3) cortical nucleus following injection of $\mathrm{Cl}$ in hamster 102 . A. cresyl violet. B. darkfield photomicrograph of coronal section in (A). Note accumulation of label at junction of $\mathrm{C} 3$ and AHA. Compare to Figure 3J. 


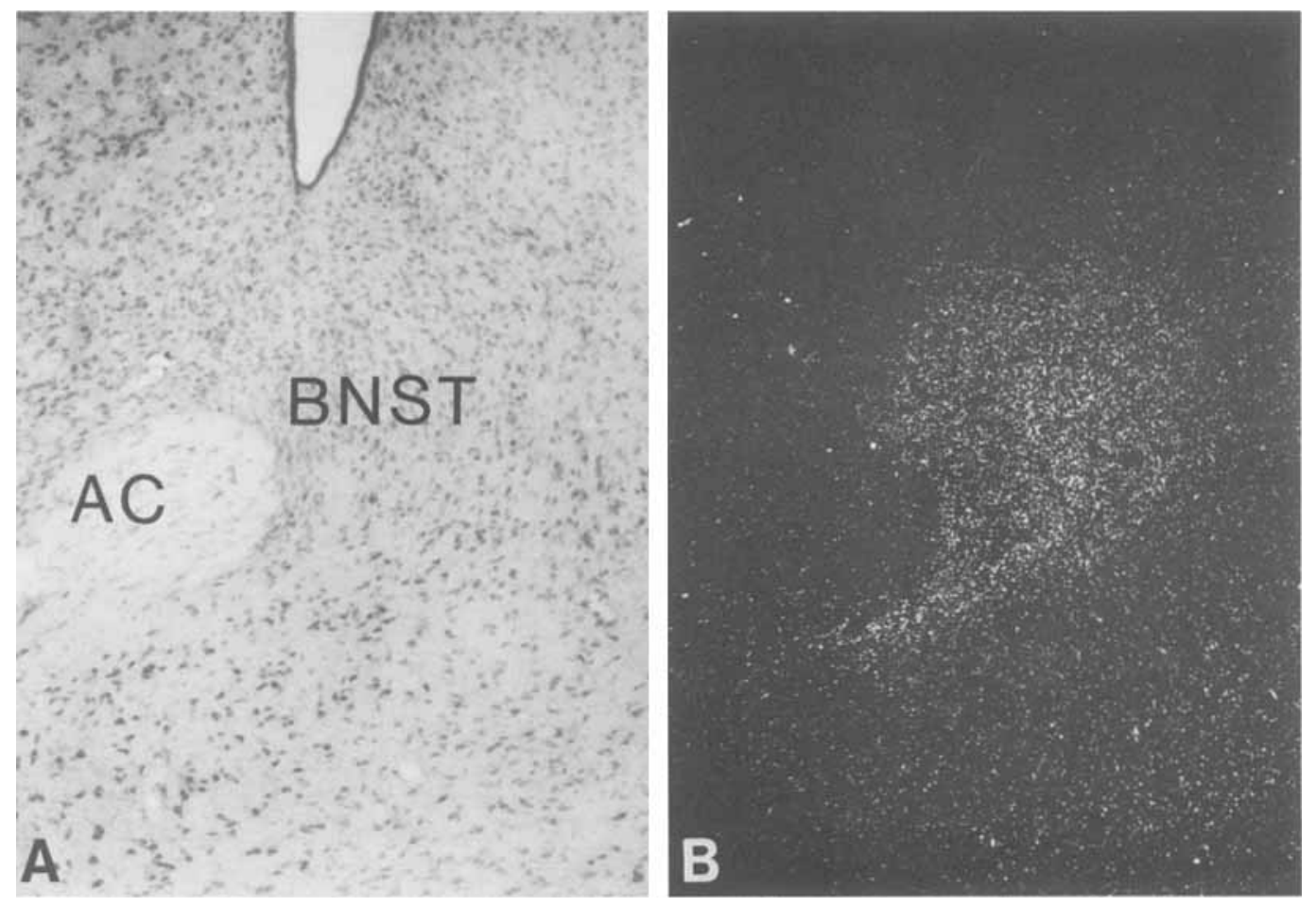

Fig. 6. Bed nucleus of the stria terminalis (BNST) following injection of tritiated proline into C1 in animal 102. A cresyl violet. B. darkfield photomicrograph of coronal section in (A). Compare to Figure 2D.

$\mathrm{C} 1$ and $\mathrm{C} 2$ in the plexiform layer of the ventral subiculum provide indirect input to VMH. Swanson and Cowan ('77) have demonstrated that the origin of the medial cortico-hypothalamic tract to the shell of the VMH is in the ventral subiculum rather than the hippocampus. Thus, VMH receives both direct and indirect input from olfactory areas of the corticomedial amygdala, as well as direct projections from the vomeronasal amygdala.

\section{Interactions of the olfactory and vomeronasal systems}

It is well established that two separate olfactory systems exist from the receptors to the terminals of the secondary neurons in the piriform lobe. In the vomeronasal system most of these secondary neurons are in the vomeronasal amydgala, which projects heavily to areas receiving projections from $\mathrm{AOB}$. In addition, $M$, the amygdaloid nucleus most implicated in sexual behavior (Harris and Sachs, '75; Lehman et al., '78), projects to the medial preoptic area and medial hypothalamus, areas that have been implicated in sexual behavior in rodents (Heimer and Larsson, '67; Giantonio et al., '70). In contrast, extrinsic olfactory amygdala projections are to tertiary olfactory areas, plus the ventral subiculum. Although the concept of a dual olfactory system is superficially supported by these projections, the separateness between these two systems is not absolute, primarily because projections from the olfactory amygdala terminate in areas that also receive vomeronasal input. First, olfactory and vomeronasal influences converge onto the core of the ventromedial nucleus of the hypothalamus. Second, olfactory processing may affect vomeronasal areas by intra-piriform lobe connections. The piriform cortex, a major olfactory terminal area, projects to the endopiriform nucleus, which in turn sends fibers that terminate in M and C3 (Krettek and Price, '78b). C2 also projects to the endopiriform nucleus in rat and cat (Krettek and Price, $78 \mathrm{~b}$ ) and hamster. Further intra-amygdaloid projections from $\mathrm{C} 1$ and $\mathrm{C} 2$ go directly to $\mathrm{C3}$ in rat and cat (Krettek and Price, '78b) and 

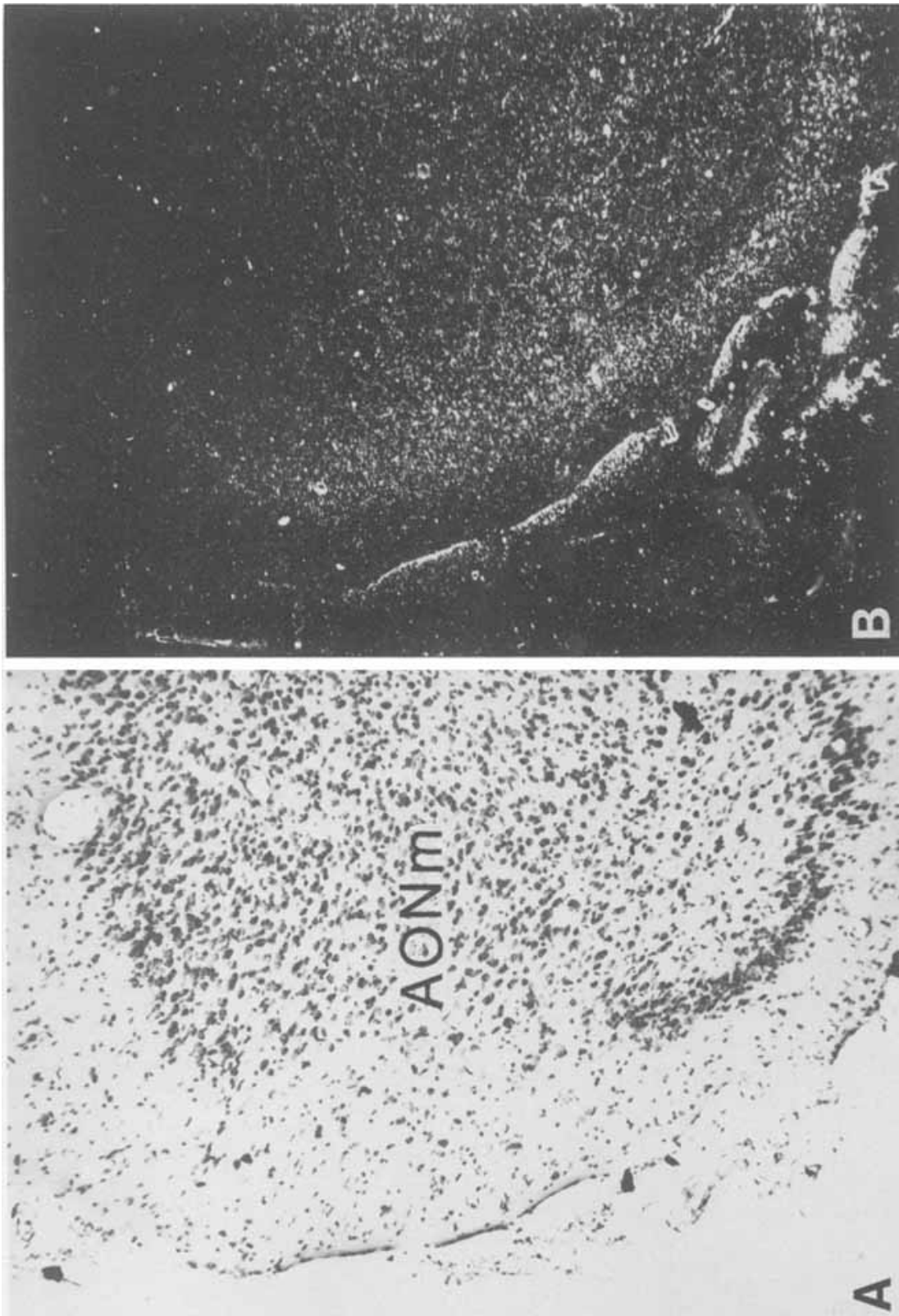

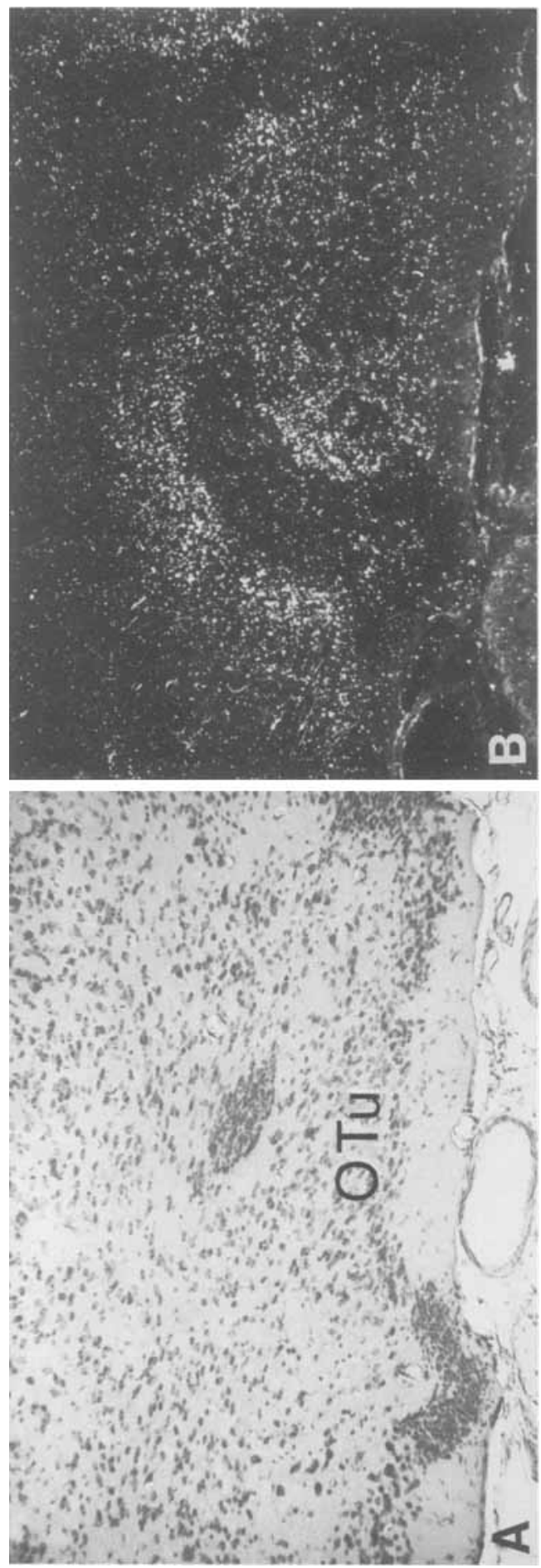

hamster, and from $\mathrm{C} 1$ and $\mathrm{C} 2$ to $\mathrm{M}$ in hamster. Therefore, influences from the main olfactory bulb may be integrated with those from the accessory olfactory bulb at the level of the amygdala in two ways: (a) by way of intrinsic connections from the olfactory amygdala to the vomeronasal amygdala, and (b) from the olfactory cortex and $\mathrm{C} 2$ projections to the endopiriform nucleus, and from this nucleus to $\mathrm{M}$ and C3 (Krettek and Price, '78b). Because $M$ and $\mathrm{C} 3$ are reciprocally connected, information reaching one component of the vomeronasal amygdala may be processed by one nucleus and then projected to the other. By these intra-piriform lobe connections, olfactory inputs influence both components of the vomeronasal amygdala.

It may be significant that no reciprocal projections were found from vomeronasal to olfactory areas. The olfactory system may therefore be affecting motivated behaviors and physiological processes in which the vomeronasal system plays a major role, while other functions are purely olfactory in nature. These latter functions may be related to learned responses processed through the olfactory systems connections with the medial and lateral prefrontal cortical areas.

\section{ACKNOWLEDGMENTS}

The authors would like to thank William Brudon for his assistance with the illustrations and Bonita Johnson for typing the manuscript.

This research was supported in part by NRSA grant T32-MH14279 to G.A.K., Rackham dissertation grant to G.A.K. from Horace H. Rackham School of Graduate Studies University of Michigan, and NIH grant RO1NS14071 to S.S.W.

\section{LITERATURE CITED}

Broadwell, R. D. (1975a) Olfactory relationships of the telencephalon and diencephalon in the rabbit. I. An autoradiographic study of the efferent connections of the main and accessory olfactory bulb. J. Comp. Neurol., 163:329-346.

Broadwell, R. D. (1975b) Olfactory relationships in the telencephalon and diencephalon in the rabbit. II. An autoradiographic and horseradish peroxidase study of the efferent connections of the anterior olfactory nucleus. $J$. Comp. Neurol., 164:389-410.

Fig. 8. Label in olfactory tubercle (OTu) after injection of $\mathrm{C2}$ in animal 93 . A. cresyl violet. B. darkfield photomicrograph of coronal section in (A). Compare to Figure 2B, C. 
Davis, B. J., F. Macrides, W. M. Youngs, S. P. Schneider, and D. L. Rosen (1978) Efferents and centrifugal afferents of the main and accessory olfactory bulbs in the hamster. Brain Res. Bull., 3:59-72.

de Olmos, J., H. Hardy, and L. Heimer (1978) The afferent connections of the main and accessory olfactory bulb formations in the rat: An experimental HRP-study. J. Comp. Neurol, $181: 213-243$.

de Olmos, J. S., and W. R. Ingram (1972) The projection field of the stria terminalis in the rat brain. An experimental study. J. Comp. Neurol., 146:303-331.

Devor, M. (1973) Components of mating dissassociated by lateral olfactory tract transection in male hamster. Brain Res., 64:437-441.

Giantonio, G. W., N. L. Lund, and A. A. Gerall (1970) Effect of diencephalic and rhinencephalic lesions on the male rat's sexual behavior. J. Comp. Phys. Psychol., 73:38-46.

Haberly, L. B., and J. L. Price (1978a) Association and commissural fiber systems of the olfactory cortex of the rat. I. Systems originating in the piriform cortex and adjacent areas. J. Comp. Neurol., 178:711-739.

Haberly, L. B., and J. L. Price (1978b) Association and commissural fiber systems of the alfactory cortex of the rat. II. Systems originating in the olfactory peduncle. J. Comp. Neurol., 181:781-807.

Harris, V. S., and B. D. Sachs (1975) Copulatory behavior in male rats following amygdaloid lesions. Brain Res., 86:514-518.

Heimer, L., and K. Larsson (1967) Mating behavior of male rats after olfactory bulb lesions. Phys. Behav., 2:207-209.

Kevetter, G. A., and S. S. Winans (1980) Connections of the corticomedial amygdala in the golden hamster. I. Efferents of the "vomeronasal amygdala." J. Comp. Neurol. 197:81-98.

Krettek, J. E., and J. L. Price (1974a) Connections of the amygdala with the bed nucleus of the stria terminalis and the hypothalamus in the rat and cat. Soc. Neurosci. Prog. Abst., 370.

Krettek, J. E., and J. L. Price (1974b) A direct input from the amygdala to the thalamus and the cerebral cortex. Brain Res., 67:169-174.

Krettek, J. E., and J. L. Price (1974c) Projections from the amygdala to the perirhinal and entorhinal cortices and the subiculum. Brain Res., 71:150-154.

Krettek, J. E., and J. L. Price (1977a) Projections from the amygdaloid complex to the cerebral cortex and thalamus in the rat and cat. J. Comp. Neurol., 172:687-722.
Krettek, J. E., and J. L. Price (1977b) Projections from the amygdaloid complex and adjacent olfactory structures to the entorhinal cortex and to the subiculum in the rat and cat. J. Comp. Neurol., 172:723-752.

Krettek, J. E., and J. L. Price (1978a) Amygdaloid projections to subcortical structures within the basal forebrain and brainstem in the rat and cat. J. Comp. Neurol., 178:225-254.

Krettek, J. E., and J. L. Price (1978b) A description of the amygdaloid complex in the rat and cat with observations on intra-amygdaloid axonal connections. J. Comp. Neurol., 178:255-280.

Lammers, H. J. (1972) The neural connections of the amygdaloid complex in mammals. In: The Neurobiology of the Amygdala. B. Eleftheriou, ed. Plenum Press, New York, pp. 123-144.

Lehman, M. N., G. A. Kevetter, and J. B. Powers (1978) Sexual and aggressive behavior in male hamsters after lesions of the corticomedial amygdala. Soc. Neurosci. Prog. Abst., 251.

McBride, R. L., and J. Sutin (1977) Amygdaloid and pontine projections to the ventromedial nucleus of the hypothalamus. J. Comp. Neurol., 174:377-396.

Raisman, G. (1972) An experimental study of the projection of the amygdala to the accessory olfactory bulb and its relationship to the concept of a dual olfactory system. Exp. Brain Res., 14:395-408.

Scalia, F., and S. S. Winans (1975) The differential projections of the olfactory bulb and accessory olfactory bulb in mammals. J. Comp. Neurol., 161:31-56.

Scalia, F., and S. S. Winans (1976) New perspectives on the morphology of the olfactory system: Olfactory and vomeronasal pathways in mammals. In: Mammalian Olfaction, Reproductive Processes, and Behavior. R. L. Doty, ed. Academic Press, New York.

Swanson, L. W. and W. M. Cowan (1977) An autoradiographic study of the efferent connections of the hippocampal formation in the rat. J. Comp. Neurol., 172:49-84.

Winans, S. S., and F. Scalia (1970) Amygdaloid nucleus: New afferent input from the vomeronasal organ. Science, 170:330-332.

Winans, S. S., and J. B. Powers (1977) Olfactory and vomeronasal deafferentation of male hamsters: Histological and behavioral analyses. Brain Res., 126:325-344. 